\title{
A Remark Concerning the Solution of the Dirichlet Problem by Finite Differences
}

\author{
By Bernard Epstein
}

In the classic paper [1] it is proven that the mesh-functions obtained by solving a discrete analogue of the Dirichlet problem converge, as the mesh-width approaches zero, to a harmonic function which solves the Dirichlet problem in a somewhat generalized sense. More precisely, let the function $f$ be continuously differentiable in a bounded plane domain $G$ and continuous in the closure $\bar{G}$ of $G$, and let the Dirichlet integral

$$
D(f)=\iint_{G}\left[f_{x}^{2}+f_{y}^{2}\right] d x d y
$$

be finite. Then the finite-difference method presented in the aforementioned paper is shown (under suitable assumptions concerning the smoothness of the boundary) to furnish a function $u$ harmonic in $G$ whose Dirichlet integral $D(u)$ is finite (and not greater than $D(f)$ ), and it is shown that $u$ agrees with $f$ on the boundary $\partial G$ in the sense that for all sufficiently small values of $\epsilon$ the inequality

$$
\iint_{s_{\epsilon}}(u-f)^{2} d x d y \leqq \gamma \epsilon^{2}
$$

holds, where $S_{\epsilon}$ denotes the portion of $G$ consisting of those points whose distance from $\partial G$ is less than $\epsilon$ and $\gamma$ denotes some positive number independent of $\epsilon$. The authors indicate, without supplying the details, that $u$ agrees with $f$ on $\partial G$ in the more elementary sense that the function $u-f$, initially defined only in $G$, becomes continuous in $\bar{G}$ if defined to vanish everywhere on $\partial G$. In this brief note we present a proof of this fact, thus showing, without reference to any other method of treating the Dirichlet problem, that the finite-difference method provides an existence proof for the "conventional" formulation of the Dirichlet problem as well as an effective procedure for computing the solution.

The proof is accomplished by establishing two lemmas. The first of these is a strengthened version of the inequality (2).

$$
\text { Lemma 1. } \lim _{\epsilon \rightarrow 0} \epsilon^{-2} \iint_{s_{\epsilon}}(u-f)^{2} d x d y=0 .
$$

Proof. First we establish (3) in the particular case that $G$ is the unit disc, $x^{2}+y^{2}<1$. Let $v=u-f$ and let two points with polar coordinates $(r, \theta)$, $(R, \theta), r<R<1$, be selected. Then

$$
v(R, \theta)-v(r, \theta)=\int_{r}^{R} v_{\rho}(\rho, \theta) d \rho=\int_{r}^{R} v_{\rho}(\rho, \theta) \rho^{1 / 2} \cdot \rho^{-1 / 2} d \rho .
$$

Applying the Schwarz inequality and assuming that $r>\frac{1}{2}$, we obtain

$$
[v(R, \theta)-v(r, \theta)]^{2} \leqq\left(\int_{r}^{R} v_{\rho}^{2} \rho d \rho\right)\left(\int_{r}^{R} \rho^{-1} d \rho\right) \leqq 2(1-r) \int_{r}^{1} v_{\rho}^{2} \rho d \rho .
$$

Received August 3, 1961. 
Integrating over $\theta$ and setting $\epsilon=1-r$, we obtain

$$
\begin{array}{r}
\int_{0}^{2 \pi}[v(R, \theta)-v(r, \theta)]^{2} d \theta \leqq 2 \epsilon \iint_{s_{\epsilon}} v_{\rho}{ }^{2} d x d y \leqq 2 \epsilon \iint_{s_{\epsilon}}\left[v_{x}{ }^{2}+v_{y}{ }^{2}\right] d x d y \\
\leqq 2 \epsilon D(v) .
\end{array}
$$

Since $D(f)$ and $D(u)$ exist, $D(v)$ also exists, so the left side of (6) must approach zero with $\epsilon$. It then follows, by a familiar argument, that $\lim _{\epsilon \rightarrow 0} \int_{0}^{2 \pi} v^{2}(r, \theta) d \theta$ exists. If this limit, which we denote by $C$, were positive, the inequality

$$
\int_{0}^{2 \pi} v^{2}(r, \theta) d \theta>\frac{1}{2} C
$$

would hold for all sufficiently small $\epsilon$. Integrating this inequality, we would obtain, in contradiction with (2),

$$
\iint_{s_{\epsilon}} v^{2}(r, \theta) r d r d \theta>\frac{1}{2} C \int_{1-\epsilon}^{1} r d r=\frac{1}{4} C \epsilon(2-\epsilon) .
$$

Having thus established that $C=0$, we return to (6), let $R \rightarrow 1$ (keeping $r$ momentarily fixed), and thus obtain, by an obvious application of the triangle inequality,

$$
\int_{0}^{2 \pi} v^{2}(r, \theta) d \theta \leqq 2 \epsilon D_{\epsilon}(v)
$$

where $D_{\epsilon}(v)=\iint_{s_{t}}\left[v_{x}{ }^{2}+v_{y}{ }^{2}\right] d x d y$. Integrating and noting that $D_{\epsilon}(v)$ is a decreasing function of $r(=1-\epsilon)$, we obtain

$$
\iint_{s_{\epsilon}} v^{2} d x d y \leqq 2 \epsilon D_{\epsilon}(v) \int_{1-\epsilon}^{1} \rho d \rho=\epsilon^{2}(2-\epsilon) D_{\epsilon}(v) .
$$

Since $D_{\epsilon}(v)$ approaches zero with $\epsilon,(9)$ implies (3).

For a more general domain with sufficiently smooth boundary it is readily seen that the same argument may be applied by introducing a coordinate system whose coordinate curves are the normals to the boundary and their orthogonal trajectories.

Before stating the second lemma, we introduce the following terminology (cf. [2, p. 481]). A $\dot{D}$-function (defined in a domain $G$ ) is one that is continuously differentiable in $G$ and has compact support, i.e., there exists $\epsilon>0$ such that the function vanishes throughout $S_{\epsilon}$. A $D^{\circ}$-function, say $v$, is one which is continuously differentiable in $G$ and can be approximated by $D$-functions in the sense that there exists a sequence $\left\{v_{n}\right\}$ of $\dot{D}$-functions such that

$$
\lim _{n \rightarrow \infty} \iint_{G}\left(v-v_{n}\right)^{2} d x d y=0, \lim _{n \rightarrow \infty} D\left(v-v_{n}\right)=0 .
$$

Lemma 2. $v(=u-f)$ is a $D_{\text {-function. }}$

Proof. As in the proof of the previous lemma, it suffices to confine attention to the case that $G$ is the unit disc. For each positive integer $n$ we define the func- 
tions $g_{n}$ and $h_{n}$ as follows:

$$
g_{n}=\left\{\begin{array}{cc}
1 & 0 \leqq r \leqq 1-\frac{1}{n} \\
\cos ^{2} n \pi\left(1-r-\frac{1}{n}\right), & 1-\frac{1}{n} \leqq r \leqq 1-\frac{1}{2 n} \\
0 & r \geqq 1-\frac{1}{2 n}
\end{array}\right\}, h_{n}=1-g_{n} .
$$

Then the functions $v_{n}=v g_{n}$ are obviously $\dot{D}$-functions, and we establish the two parts of (10) by the following arguments.

$$
\iint_{G}\left(v-v_{n}\right)^{2} d x d y=\iint_{G}\left(v h_{n}\right)^{2} d x d y
$$

$$
\begin{array}{r}
=\iint_{s_{1 / n}}\left(v h_{n}\right)^{2} d x d y \leqq \iint_{s_{1 / n}} v^{2} d x d y \rightarrow 0 ; \\
D\left(v-v_{n}\right)=D\left(v h_{n}\right)=D_{1 / n}\left(v h_{n}\right) \leqq D_{1 / n}\left(v h_{n}\right) \\
\left.+\iint_{s_{1 / n}}\left[v_{x} h_{n}-v h_{n, x}\right)^{2}+\left(v_{y} h_{n}-v h_{n, y}\right)^{2}\right] d x d y \\
=2 \iint_{s_{1 / n}}\left[h_{n}{ }^{2}\left(v_{x}{ }^{2}+v_{y}{ }^{2}\right)+v^{2}\left(h_{n, x}^{2}+h_{n, y}^{2}\right)\right] d x d y .
\end{array}
$$

Since $h_{n}{ }^{2} \leqq 1$ and $h_{n, x}^{2}+h_{n, y}^{2} \leqq n^{2} \pi^{2}$, we obtain

$$
D\left(v-v_{n}\right) \leqq 2 D_{1 / n}(v)+2 n^{2} \pi^{2} \iint_{s_{1 / n}} v^{2} d x d y .
$$

$D_{1 / n}(v)$ approaches zero with increasing $n$, and the same is true of the remaining term, by Lemma 1 . Thus the present lemma is proven.

The desired result concerning the boundary behavior of $u$ now follows immediately from the following theorem (which is a particular case of a theorem proven in [2, p. 495-7]): Let $u$ be harmonic in a bounded domain $G$, let $f$ be continuous in

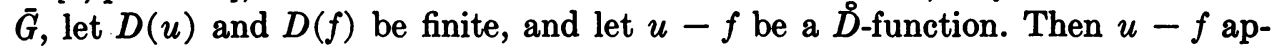
proaches zero at the boundary if the latter satisfies certain mild conditions.

Petrovsky [3] has presented a proof that the boundary values are assumed. His proof, which also appears in [4, p. $186 \mathrm{ff}$.], does not make use of (2) at all. However, the Petrovsky proof does not appear to extend readily to more general elliptic equations, whereas the proof of (2) presented in [1] and the argument presented here can be suitably modified so as to apply to other equations.

\section{Graduate School of Science}

Yeshiva University

New York 33, N. Y.

1. R. Courant, K. Friedrichs \& H. Levy, "t'ber die partiellen Differenzengleichungen der mathematischen Physik," Math. Ann., v. 100, 1928, p. 32-74.

2. R. Courant \& D. Hilbert, Methoden der mathematischen Physik, v. 2, J. Springer, Berlin, 1937.

3. I. G. Petrovskx, "New proof of the existence of a solution of Dirichlet's problem by the method of finite differences," Uspehi Mat. Nauk., v. 8, 1941, p. 161-170.

4. J. D. TAmarkin \& W. Feller, Partial Differential Equations, Mimeographed lecture notes, Brown University, Providence, 1941. 